\title{
Unusual skin sepsis due to a strain of multiresistant Staphylococcus aureus
}

\author{
JOY HARRISON AND R. W. LACEY \\ From the Departments of Microbiology, Frenchay Hospital and University of Bristol
}

SYNOPSIS A strain of Staphylococcus aureus resistant to as many as nine distinct antibiotics has shown unusally high virulence as indicated by its ability to cause an epidemic of furunculosis amongst hospital nurses.

About twenty years ago much cutaneous and wound sepsis resulted from the epidemic spread of the type $80 / 81$ strain of Staphylococcus aureus. This strain was often resistant to penicillin, streptomycin, and tetracycline but to no other antibiotics and appeared to be exceptionally virulent (eg, Rountree and Freeman, 1955). During subsequent years the incidence of this type in hospitals has declined, and strains of phage group III which are often resistant to more than three antibiotics (eg, Jevons, John, and Parker, 1966) have increased. There has probably also been a reduction in the number of incidents of sepsis by hospital staphylococci; this is probably due in part to a decline in virulence of the organism (Williams, 1971). Thus the acquisition of resistance to many antibiotics may be associated with a decline in virulence. Theoretical considerations support this assertion (Lacey, 1973) and there is some experimental evidence that plasmid carriage is associated with a reduction in virulence (Lacey and Chopra, 1974). Although some serious infections have been due to multi-resistant strains of Staphylococcus aureus during the last decade (Eriksen, 1967; Benner and Kayser, 1968; Hallander and Laurell, 1971), these were mainly in patients who were already suffering from serious trauma or debilitating disease. This apparent invasiveness of the hospital staphylococcus could be due to decreased host resistance, possibly augmented by the use of immunosuppressive agents or cytotoxic drugs. Such multiresistant staphylococci have produced little primary skin sepsis (Parker and Hewitt, 1970), so that the overall picture is still that of relative avirulence associated with resistance to many antibiotics.

However, during 1973 multiresistant isolates of Received for publication 5 August 1974.
Staphylococcus aureus have been implicated in instances of severe primary skin sepsis in otherwise healthy individuals in a district general hospital. This suggests that multi-resistant strains may be fully virulent. This report describes the outbreak.

\section{The Outbreak}

The episode occurred in a district general hospita? of 560 beds which also contained the regionat. neurosurgery, thoracic surgery, plastic surgery and burns units The last was a single storey, L-shaped building constructed in 1930 for the treatment of tuberculosis, and was about two hundred yards from the rest of the hospital which was composed of single-storey wards connected by two long corridors.

On 16 June 1973 a patient was admitted to the hospital with a badly crushed hand for which a partial amputation and reconstruction was done on the day of admission. This involved a fullthickness skin flap from the chest wall, to which the hand remained attached for three weeks. On 26 June, 10 days after the operation, the area on the chest wall from which the pedicle had been fashioned showed gross sepsis, with copious purulent discharge and inflammation extending about $2 \mathrm{~cm}$ on to the surrounding intact skin. The pedicle and recipient areas were also tense and red. From the donor area, the pedicle, and hand Staphylococcus aureus type 77 was grown. The organism had not been identified on the ward for the previous three months, although it had been isolated occasionally from this and other departments of the hospital during the previous two years. On 12 September, a nurse working on the ward developed pustules on both forearms and a week later a boil on the back of one hand.

Staphylococcus aureus type 77 was grown from 


\begin{tabular}{|c|c|c|c|c|}
\hline Date of Isolation & Index No. & Source & $\begin{array}{l}\text { Phage Typing } \\
\text { Pattern (RTD) }\end{array}$ & Antibiogram \\
\hline $\begin{array}{l}14.9 .73 \\
14.9 .73 \\
26.9 .73 \\
1.10 .73 \\
2.10 .73 \\
29.10 .73 \\
5.11 .73 \\
8.11 .73 \\
13.2 .73\end{array}$ & $\begin{array}{l}\text { D167 } \\
\text { D167 } \\
\text { D168 } \\
\text { D176 } \\
\text { D179 } \\
\text { D218 } \\
\text { D237 } \\
\text { D252 } \\
\text { D312 }\end{array}$ & $\begin{array}{l}\text { Pustules of forearm, nurse } \mathbf{K} \\
\text { Boil on hand, nurse K } \\
\text { Anterior nares, nurse K } \\
\text { Axillary boils, nurse R } \\
\text { Pustules on face, nurse A } \\
\text { Nose swab, nurse G } \\
\text { Pustules on arm, nurse C } \\
\text { Boil of nose, nurse P } \\
\text { Pustule on arm, nurse B }\end{array}$ & $\begin{array}{l}77 \\
77 \\
77 \\
77 \\
77 \\
77 \\
77 \\
77 \\
77\end{array}$ & $\begin{array}{l}\text { S T Su M N P E L Sp } \\
\text { S T Su M N E L Sp } \\
\text { S T Su M N P E L Sp } \\
\text { S T Su P } \\
\text { S T Su M P } \\
\text { S T Su M P } \\
\text { S T Su M P } \\
\text { S T Su M P } \\
\text { S T Su M P E L Sp }\end{array}$ \\
\hline
\end{tabular}

Table Properties of some cultures of Staphylococcus aureus associated with epidemic infection of nurses

${ }^{1} \mathrm{~S}=$ resistance to streptomycin (MIC $>1 \mathrm{mg} / \mathrm{ml}$ ); $\mathrm{T}=$ to tetracycline (approx $100 \mu \mathrm{g} / \mathrm{ml}$ ), $\mathrm{Su}=$ to sulphamethoxazole (approx $200 \mu \mathrm{g} / \mathrm{ml}$ ), $\mathrm{N}=$ to neomycin (approx $25 \mu \mathrm{g} / \mathrm{ml}$ ), $\mathbf{E}=$ to spectinomycin (approx $5 \mathrm{mg} / \mathrm{ml}$ ), $\mathbf{P}=$ production of penicillinase, $\mathbf{M}=$ heterogenous reaction to methicillin. MICs determined by the method of Lacey et al (1974)

each of these sites and also from a nasal swab. During the next eight weeks three patients with extensive burns became colonized with the strain, one nurse developed axillary boils, another nurse a nasal boil, and two other nurses had pustules on the forearms. The same organisms were also carried in the nose of one nurse, and a further nurse had a widespread pustular eruption from which the staphylococcus was cultured. The pustules were mainly on the skin areas immersed when saline baths were given to patients with burns. Some properties of the strain isolated from the lesions are shown in the table.

\section{Investigation and Treatment}

Nasal and wound swabs were taken from all the staff and patients on the ward. Further admissions to the unit and the transfer of staff to other wards was stopped. As many patients as possible were discharged home and remaining patients with negative swabs were transferred to another wing of the unit. Four severely ill patients who had bacteriophage type 77 Staphylococcus aureus remained and were nursed in isolation. Their soiled linen was deposited in plastic bags at the bedside, paper gowns and masks were worn on entering and discarded on leaving. Visitors were allowed. When staff became due for leave, clearance swabs were taken and only after these had been proved negative were they transferred to the 'clean' wing or other wards.

Two nurses ( $R$ and $K$ ) who had boils went on sick leave, and the other affected nurses remained on duty but worked only in the infected wing. Nurse $\mathrm{R}$ suffered from a crop of axillary boils that lasted several weeks. Not being resident she was treated initially by her general practitioner with penicillin and then with flucloxacillin. She was later referred to the hospital Casualty Department, where the axillary boils were incised. She was the only affected nurse to receive antibiotics and the only one who became a nasal carrier after clinical recovery of the lesions. Nasal carriage in other patients was uncommon, even during the presence of pustular lesions.

The following personal regime was carried out for 14 days by all those affected by Staphylococcus aureus type 77, including patients as far as their clinical condition allowed:-(1) $1 \%$ chlorhexidine cream was applied to the anterior nares thrice daily. (2) A daily bath in about 15 gallons of water containing $30 \mathrm{ml}$ chlorhexidine concentrate was taken. (3) Soap containing $2 \%$ hexachlorophane and dusting powder containing $0.33 \%$ hexachlorophane were used. (4) Hair was washed thrice weekly and rinsed in 1 in 2000 aqueous chlorhexidine.

Forty-eight hours after the regime ended, clearance swabs were taken from the nose and any previously infected areas. Because the chlorhexidine nasal cream caused soreness, the same strength chlorhexidine prepared as an ointment was later substituted. There were no complaints of cracking or soreness from its use. In an effort to economize, a povidone-iodine sachet has now been used in the bath and a chlorhexidine plus cetrimide sachet for hair washing.

Two months after the first nurse reported sick, the last patient whose burns were infected with type 77 Staphylococcus aureus was discharged. Final clearance swabs were then taken from relevant staff, the ward was springcleaned, redecorated, and re-opened about three months after the episode started. The epidemic strain has not reappeared in the ward since then.

\section{Discussion}

It is difficult to quantify the virulence of many bacteria towards man. Nevertheless, the production of primary skin lesions in otherwise healthy individuals must indicate considerable pathogenic powers of these hospital staphylococci. The mech- 
anism of this virulence is obscure. Enhanced virulence rarely, if ever, arises by mutation in vitro, and there is apparently no instance of such a mutation occurring in vivo. Since virulence in Staphylococcus aureus probably results from many factors (Elek, 1959); the region of the genome that determines virulence is likely to be relatively large. Thus the acquisition of virulence could well result from the transfer of the relevant genes from another staphylococcus to these cultures. This mechanism would be analogous to the conversion of nontoxigenic to toxigenic Corynebacterium diphtheriae due to acquisition of prophage. It is necessary to postulate only one transfer event, since each of the septic incidents was probably due to staphylococci that were all essentially the same strain. Changes in the antibiotic sensitivity in this strain were almost certainly due to variations in the carriage of plasmids (Lacey, Lewis, and Harrison, 1974). Consistent with the proposal that enhancement of virulence had resulted from acquisition of a relatively large piece of DNA is the finding that in vitro the decrease of virulence associated with carriage of several plasmids was probably caused by the loss of such DNA (Lacey and Chopra, 1974).

It is premature to predict that hospital staphylococci will in general become both fully virulent and resistant to many antibiotics. But the threat exists.

References

Benner, E. J., and Kayser, F. H. (1968). Growing clinical significance of methicillin-resistant Staphylococcus aureus. Lancet, 2 , 741-744.

Elek, S. D. (1959). Staphylococcus pyogenes and its Relation to Disease. Livingstone, Edinburgh.

Eriksen, K. R. (1967). Paper presented at the Vth International Congress of Chemotherapy, Vienna.

Hallander, H. O., and Laurell, G. (1971). Epidemiological and clinical aspects of methicillin resistance and enterotoxin production in Staphylococcus aureus. Ann. N.Y. Acad. Sci., 182, 98-105.

Jevons, M. P., John, M., and Parker, M. T. (1966). Cultural characters of a newly recognized group of hospital staphylococci. $J$. clin. Path., 19, 305-312.

Lacey, R. W. (1973). Genetic basis, epidemiology and future significance of antibiotic resistance in Staphylococcus aureus: A review. J. clin. Path., 26, 899-913.

Lacey, R. W., and Chopra, I. (1974). Effect of plasmid carriage on the virulence of Staphylococcus aureus. (Submitted to J. med. Microbiol.)

Lacey, R. W., Lewis, E., and Harrison, J. (1974). Variation in the properties of a type 77 Staphylococcus aureus isolated over 3 months from a hospital. J. med. Microbiol., in press.

Parker, M. T., and Hewitt, J. H. (1970). Methicillin resistance in Staphylococcus aureus. Lancet, 1, 800-804.

Rountree, P. M., and Freeman, B. M. (1955). Infections caused by a particular 'phage type of Staphylococcus aureus. Med J. Aust. 2, 157-161.

Williams, R. E. O. (1971). Changes in the virulence and antibiotic resistance of Staphylococcus aureus in bacterial infections: changes in their causative agents, trends, and possible basis Edited by M. Finland, W. Marget, and K. Bartmann, p. 990 Springer, Berlin. 